PALEO

Revue d'archéologie préhistorique

15 | 2003

Varia

\title{
Les feuilles de laurier de Volgu (Saône-et-Loire) : une énigme en partie résolue?
}

Volgu laurel leaf points: an enigma partially resolved?

\section{Thierry Aubry, Jean-Baptiste Peyrouse et Bertrand Walter}

\section{(2) OpenEdition}

\section{Journals}

Édition électronique

URL : http://journals.openedition.org/paleo/1308

DOI : $10.4000 /$ paleo.1308

ISSN : 2101-0420

Éditeur

SAMRA

\section{Édition imprimée}

Date de publication : 1 décembre 2003

Pagination : 251-254

ISSN : $1145-3370$

\section{Référence électronique}

Thierry Aubry, Jean-Baptiste Peyrouse et Bertrand Walter, « Les feuilles de laurier de Volgu (Saône-etLoire) : une énigme en partie résolue ?», PALEO [En ligne], 15 | 2003, mis en ligne le 03 août 2010, consulté le 10 décembre 2020. URL : http://journals.openedition.org/paleo/1308 ; DOI : https:// doi.org/10.4000/paleo.1308

Ce document a été généré automatiquement le 10 décembre 2020.

\section{(c) $($ () $\odot$}

PALEO est mis à disposition selon les termes de la licence Creative Commons Attribution - Pas d'Utilisation Commerciale - Pas de Modification 4.0 International. 


\section{Les feuilles de laurier de Volgu (Saône-et-Loire) : une énigme en partie résolue?}

Volgu laurel leaf points: an enigma partially resolved?

Thierry Aubry, Jean-Baptiste Peyrouse et Bertrand Walter

Dans le cadre de notre étude des grandes feuilles de laurier du site solutréen de production des Maîtreaux (Aubry et al. 1998, 2003 s.p.; Aubry et Walter 2003), nous avons entrepris de comparer les différents ensembles lithiques où ces outils sont présents. Nous nous sommes particulièrement intéressés aux feuilles de Volgu (Saôneet-Loire) qui sont parmi les rares à avoir été découvertes entières. Ces pointes foliacéessont à l'origine du type J, proposé par P. Smith (1966). Afin de comparer leurs matériaux et leurs modalités de fabrication, nous avons étudié les 13 pièces présentées au Musée de Chalon-sur-Saône $e^{1}$ et l'exemplaire exposé au Musée des Antiquités Nationales de Saint-Germain-en-Laye.Nous avons bénéficié des renseignements fournis par J. Pelegrin en ce qui concerne la dernière feuille de Volgu publiquement connue, conservée au British Museum.

\section{La " cache" des feuilles de Volgu et l'énigme de l'origine de leur matière première}

2 La découverte d'au moins 15 grandes feuilles de laurier ${ }^{2}$, lors du creusement d'un canal dans les alluvions de l'Arroux proche de sa confluence en rive droite de la Loire, est singulière dans le contexte solutréen. Les circonstances de la découverte faite en 1874 ont été relatées en détail dans le rapport établi par F. Chabas (1874). Celui-ci permet d'établir que les pièces se trouvaient enterrées à 1 mètre au-dessous du niveau du sol, disposées sur leur tranche, séparées par une fine couche de sédiments, dans un dépôt de limons superposéà une nappe alluviale située à 10 mètres environ de l'étiage de l'Arroux. La découverte de fragments d'une meule romaine et de céramiques, non 
positionnés en stratigraphie, ne fournit pas de renseignements sur la chronologie des dépôts. Les observations effectuées lors de la découverte indiquent qu'aucun autre objet de pierre taillée n'accompagnait les feuilles dans ce qui a été décrit comme la “ cache " de Volgu. L'indice d'une fréquentation solutréenne de la région la plus proche de Volgu est le site de Solutré, distant de $60 \mathrm{~km}$ vers l'est (fig. 1B, $\mathrm{n}^{\mathrm{o}} 8$ ).

Figure 1 - Localisation des formations géologiques dont les silex ont été comparés avec ceux des feuilles de Volgu.

Figure 1 - Location map of the geological formations used for comparison with the raw material of Volgu laurel leaf points.

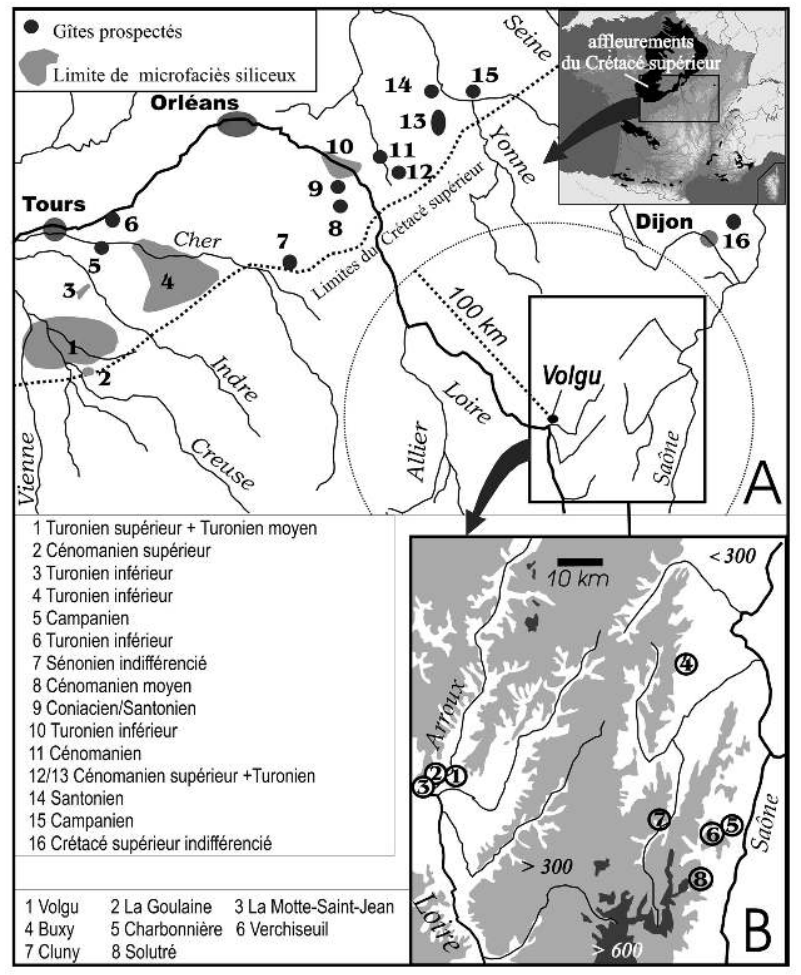

Dès la présentation du rapport par F. Chabas, la nature exceptionnelle du silex des feuilles de Volgu a été remarquée. Pour lui, il ne peut être local : “ le silex avec lequel ces lames ont été fabriquées est de l'espèce appelée pyromaque; quelques lames sont de couleur blonde et entièrement diaphanes, mais la plupart sont parsemées de tâches noirâtres, qu'on n'aperçoit qu'à l'aide de la transparence. (...) Les silex naturels de la localité n'offrent pas les mêmes conditions de texture et de dimension ". Pour A. Arcelin (1874), il n'est pas nécessaire de chercher une origine allochtone à ces silex : les gîtes de Charbonnière, dans les monts du Mâconnais (fig.1B, $\mathrm{n}^{\mathrm{0}}$ 5), recèleraient des silex de module et d'aspect macroscopique similaires qui auraient pu convenir à la taille des feuilles de Volgu. L'auteur note cependant que " tandis que le silex de Volgu ne renferme d'après M. Chabas, aucune trace de corps organisés, ceux de Charbonnières sont généralement parsemés de débris de bryozoaires, qu'on aperçoit comme des taches blanchâtres dans toute la masse du silex". D'autres auteurs envisagent une source d'approvisionnement encore plus proche. Pour J.-B. Jost (1927), le silex blond des feuilles qu'il identifie comme issu de la craie (ce qui le différencie du silex alluvionnaire jaune foncé de Volgu même), proviendrait de quelques kilomètres à peine de de la cache : à La Motte-Saint-Jean (fig. 1B, $\mathrm{n}^{0} 3$ ), notamment, ce chercheur a rencontré " plusieurs gros blocs de ce silex blond". R. 
Desbrosse et R. Horiot (1972) font les mêmes constatations. Ils évoquent La Goulaine (fig. $1 \mathrm{~B}, \mathrm{n}^{\mathrm{o}} 2$ ), à $4 \mathrm{~km}$ de Volgu, riche en "rognons d'un silex blond et diaphane, parfois parsemé de tâches noirâtres, dont certains pèsent plus de $10 \mathrm{~kg}$ ". C'est sur cette commune qu'un grand nucléus magdalénien, découvert en 1893, a été déterminé comme provenant de sources tertiaires du centre ou sud du Bassin parisien (Surmely et al. 2002). Jean Combier (2002) a étudié les vestiges lithiques gravettiens provenant du site de Solutré et noté qu'ils sont confectionnés majoritairement dans des silex d'origines régionales, distincts des feuilles de Volgu: "le silex de haute qualité de ces pièces, probablement originaire de Touraine et du Gâtinais (des étages du Turonien et Sénonien) est tout différent de celui du Mâconnais qui est opaque, souvent carrié et riche en bryozoaires; il n'existe aucun site solutréen intermédiaire qui puisse permettre d'envisager une relation entre ces deux points (Volgu et Solutré)".

\section{Nouvelles hypothèses sur l'origine du silex des feuilles de Volgu}

Lors de l'observation des feuilles de Volgu, nous avons décrit, macroscopiquement et à la loupe binoculaire, le microfaciès et le contenu paléontologique de chacune des pièces. Trois microfaciès siliceux peuvent être distingués :

- le plus fréquent (1) est de texture mudstone, homogène et à grain très fin, translucide, gris (P-71, R-73, P-73, N-73, N-92 du code des couleurs des sols de A. Cailleux), brun clair (N-67), gris rose (N-70), brun (P-30). Les feuilles montrent de rares inclusions grenues de quartz opaque, de teinte grise, entourée par une zone millimétrique plus claire, opaque, ou des tâches diffuses de teinte rouge jaune (P-59), gris rose (M-30), rouge faible (S-20) qui révèlent leur séjour dans des altérites. Deux feuilles possèdent des vestiges de cortex tellement réduits qu'ils ne fournissent pas d'information pertinente. Les deux pièces du British Museum et de Saint-Germain-en-Laye pourraient être isolées du groupe par leur teinte plus claire et leurs inclusions plus fréquentes.

- la seconde variété (2), représentée par deux pièces (l'une référencée en rouge no2 et l'autre, une base cassée, sans référence) est de même texture mudstone, à structure zonée par endroits, légèrement plus opaque, de teinte brun clair (N-67) à brun très pâle (M-75). La pièce complète possède des inclusions en quartz autour desquelles s'organise la zonation.

- le troisième groupe (3) est représenté par deux feuilles ( $n^{\circ} 10$ et $\left.n^{\circ} 5\right)$ de microfaciès distinct. Leur texture est semblable. L'une est gris foncé (T-31) avec des zones diffuses, plus claires et légèrement plus opaques, l'autre possède deux zones, l'une translucide de teinte brun rouge foncé (T-30) et l'autre opaque grise (M-30). La première conserve un petit fragment de cortex brun jaune à sa base.

Comme l'avait remarqué $\mathrm{F}$. Chabas, l'examen systématique à la loupe binoculaire n'a permis d'observer que de rares fragments de fossiles, dont plusieurs coupes et plaques d'échinides (dans les catégories 1 et 2), quelques spicules de spongiaires (1), des fragments de bryozoaires (1) et des tubes de vers (1).

Suivant les indications disponibles (Arcelin 1874 ; Rat 1987; Combier 2002 ; Surmely et al. 2002) nous avons collecté des échantillons sur les gîtes de la région de Charbonnières, Verchiseuil, Cluny et de Buxy (fig.1B), attribués à la décalcification de formations du Crétacé supérieur à partir de l'Eocène (Rat 1987). Ce sont des silex riches en silice fibreuse avec de nombreuses inclusions de quartz, de teinte gris bleu à brun pâle, se distinguant nettement des silex des feuilles de Volgu par la présence des 
fragments de bryozoaires qu'avait notés A. Arcelin. Le silex de Saint-Boil et de Buxy, dépourvu de ces fossiles, ne correspond plus à celui des feuilles de Volgu, beaucoup plus translucide. Quant aux échantillons provenant de la formation G2-e1, de la région de Volgu, ils possèdent des teintes et une structure zonée, liée à une épigénie secondaire en altérite qui ne peuvent aussi être confondues avec les matériaux des feuilles de Volgu. Il en va de même pour le silex tertiaire vacuolaire de La Motte-SaintJean, qui, pourtant, peut se présenter en gros blocs blonds translucides.

7 Le microfaciès des feuilles de Volgu se distingue donc nettement des différentes variétés disponibles localement et évoque, comme le remarque J. Combier (2002), celui des silicifications de la craie blanche de la base du Turonien inférieur du sud du bassin versant du Cher (fig. 1A, $\mathrm{n}^{\circ} 3,4$ et 6 ) dont la diffusion est attestée dans la région Centre (Aubry 1991), vers l'Auvergne, pendant tout le Paléolithique supérieur (Masson 1981; Bracco 1996; Surmely et al., 1998) et postérieurement (Thevenot 1991). Toutefois, cette hypothèse ne peut être retenue après notre examen car les feuilles de Volgu ne contiennent que de trop rares restes de bryozoaires, de spicules de spongiaires et de terriers de vers, indiquant une formation dans un milieu sédimentaire distinct.

Une recherche bibliographique des formations géologiques susceptibles de contenir des accidents siliceux formés dans un milieu sédimentaire correspondant a orienté notre prospection. Les craies du Crétacé supérieur de la région de Magny-Saint-Médard, au nord-est de Dijon (fig. 1A, $\mathrm{n}^{\circ} 16$ ), contiennent de rares nodules de silex de module et de microfaciès complètement distincts des feuilles. Après avoir éliminé plusieurs autres possibilités (fig.1A, no 1, 2, 5 et 7), en prenant la Loire comme premier axe d'orientation et en croisant les données géologiques avec l'existence de vestiges d'industries magdaléniennes taillées sur des blocs de silex de grand module, dans la région de SaintBrisson-sur-Loire (Allain, 1981), notre attention a été retenue par les silicifications du Turonien inférieur de la région de Gien (fig. $1 \mathrm{~A}, \mathrm{n}^{\mathrm{o}} 10$ ). La vérification sur le terrain nous a permis de retrouver un microfaciès proche du premier groupe des pièces archéologiques, dans les altérites formées à partir de bancs continus de silex contenus dans les biomicrites du Turonien inférieur des deux rives de la Loire autour de Gien (Gigout 1977), soit à 160 kilomètres en aval de Volgu.

Ce microfaciès est semblable à celui utilisé par les Magdaléniens de Saint-Brisson-qurLoire proche de ces gîtes (nous remercions monsieur P. Bazin pour avoir mis à disposition cette série). Le deuxième groupe n'a pas été retrouvé de manière certaine et le troisième, hétérogène nous l'avons vu, n'a pas été détecté sur le terrain même si l'hypothèse d'une modification nette de la teinte dans les altérites n'est pas à éliminer. Ces indices nous ont conduit à considérer d'autres sources possibles. Le Sénonien et le Cénomanien supérieur de la même région (fig. $1 \mathrm{~A}, \mathrm{n}^{\circ} 8$ et 9 ) ne nous ont pas révélé de silex correspondant. Ceux des craies cénomaniennes, turoniennes et sénoniennes qui affleurent entre la Loire et l'Yonne (fig. $1 \mathrm{~A}, \mathrm{n}^{\circ} 11$ à 15) ne nous ont pas révélé de microfaciès semblables au premier groupe et plus convaincants que des échantillons $d u$ Turonien inférieur de la région de Gien. Cependant les silex du Santonien récoltés à La Celle-Saint-Cyr présentent des convergences avec les caractéristiques du troisième groupe pétrographique. 


\section{Matériaux des grandes feuilles de laurier solutréennes et hypothèse sur leur mode de diffusion}

10 Si nous ne sommes pas encore en mesure de proposer une origine précise pour toutes les feuilles de Volgu, il nous paraît établi qu'elles ont été déplacées depuis plusieurs sources de la bordure méridionale du Bassin parisien. Cette hypothèse implique que les nombreux vestiges liés à leur fabrication, comparables à ceux mis en évidence sur le site des Maitreaux, doivent exister à proximité immédiate des affleurements. Les conditions géomorphologiques relevées sur ce dernier site indiquent que la prospection minutieuse $d u$ fond des vallées affluentes du réseau hydrographique principal qui ont mis au jour et déstabilisé des dalles de silex formées dans les craies, pourrait permettre de mettre en évidence des indices solutréens.

11 La découverte de groupements de pièces foliacées bifaces, sans contexte archéologique, décrits sous le terme de " cache ", a plusieurs fois été évoquée pour des vestiges attribués au Solutréen :à Volgu, à Montaud dans les Landes, à La Guitière (Vienne) et sur le site de plein airde Monte da Fainha, au sud du Portugal (Roche et al., 1968). Si à Volgu le façonnage est poussé à son stade ultime, dans les deux autres cas, il s'agit de préformes évoquant les pièces bifaciales signalées dans un tout autre contexte géographique et chronologique, en plusieurs points de l'Amérique du Nord. Ces dernières, confectionnées dans des matériaux provenant de sources distantes de plusieurs dizaines de kilomètres, ont été interprétées comme un moyen rentable de déplacement de réserves de matière première (Baker 2001).

12 Les observations que nous avons pu effectuer sur les fragments de grandes feuilles de laurier des sites du bassin versant de la Creuse et d'autres régions indiquent l'absence de stigmate de fracture d'impact en percussion lancée (contrairement aux pièces de moins de $15 \mathrm{~cm}$ ), leur réutilisation systématique après fracture accidentelle lors de l'amincissement ou fracture intentionnelle par percussion sur une face (Aubry et al. 2003). Leur fonction première, l'intérêt fonctionnel de leur grand module, leur éventuel rôle symbolique et leurs lieux d'utilisation restent encore des énigmes.

\section{BIBLIOGRAPHIE}

ALLAIN J. 1981 - Rapport de la direction Région Centre, Gallia Préhistoire, T. XXIV, fasc.2, pp. 329 363.

ARCELIN A. 1874 - Compte-rendu du contenu et des conclusions du rapport de M. Chabas. Annales de l'Académie de Mâcon, séance du 28 mai 1874, tome 12, pp. 147-154.

AUBRY T. 1991 - L'exploitation des ressources en matières premières lithiques dans les gisements solutréens et badegouliens du bassin versant de la Creuse (France), thèse de Doctorat de l'Université de Bordeaux I, 7/11/1991, 1 vol, 327 p. 
AUBRY T., WALTER B., ROBIN E., PLISSON H. et BENHABDELHADI M. 1998 - Le site solutréen de plein air des Maitreaux (Bossay-sur-Claise, Indre et Loire) : un faciès original de production lithique. Paléo ํo 10, pp. 163 - 184.

AUBRY T., WALTER B., ALMEIDA M. et NEVES M.-J. 2003 - Solutrean Laurel Leafs Points Production and Raw Material Procurement during the Last Glacial Maximum in Southern Europe: Two examples from Central France and Portugal. In: From "Coups de Poing" to Clovis: Multiple Approaches to Bifaces Variability. AAA annual Congress 2000, Philadelphia. M. SORESSI and H. DIBBLE EDS. pp. 165-182.

AUBRY T. et WALTER B. 2003 - Reconstitution des modalités d'approvisionnement et de diffusion des matières premières lithiques pendant le Paléolithique supérieur - l'apport du site solutréen et badegoulien des Maitreaux (Indre-et-Loire, France). In : Actes de la table ronde internationale, "Les matières premières lithiques en Préhistoire ", Aurillac, 20-23/06/2002. Préhistoire du Sud-Ouest, Supplément $n .95$, pp 41-50.

AUBRY T., WALTER B., ALMEIDA M., LIARD M. et NEVES M.-J. s.p. - Approche fonctionnelles des sites dits d'atelier : l'exemple des occupations solutréennes et badegouliennes du site des Maitreaux (Indre-et-Loire, France). Actes du XXVo Congrès Préhistorique de France, “Approches fonctionnelles en Préhistoire ". Nanterre, 24-26 novembre 2000.

BAKER T. 2001 - Transportation and caching Lithic material in Biface Form. http://www.ele.net/ moorehead/oldwine.htm

BRACCO J.-P. 1996 - Du site au territoire. L'occupation du sol dans les hautes vallées de la Loire et de l'Allier au Paléolithique supérieur (Massif Central). Gallia Préhistoire, t. 38, pp. 43 - 67.

CHABAS F. 1874 - Les silex de Volgu (Saône-et-Loire), rapport à la Société d'Histoire et d'archéologie de Chalons-sur-Saône. Chalon-sur-Saône. 24 p., 4 pl.

COMBIER J. 2002 - Le problème des déplacements humains, le territoire des chasseurs de Solutré. In : J. Combier et A. Montet-White (dir.) - Solutré 1968 - 1998. Paris : Société Préhistorique Française. Mémoire 30, 281 p.

DESBROSSE, R. et HORIOT, R. 1972 - Un nouveau gisement paléolithique à Rigny-sur-Arroux. Bulletin de la Société d'Histoire Naturelle d'Autun, septembre 1972, n 63, pp. 11-28.

GIGOUT M. 1977 - Carte géologique de la France au 1/ 50 000, Gien, XXIV - 21.

JOST J. B. 1927 - La Préhistoire dans la Région Digoinaise. Digoin. 37 p.

MASSON A. 1981 - Pétroarchéologie des roches siliceuses : intérêt en préhistoire; Thèse de l'Université de Lyon, I, $101 \mathrm{p}$.

RAT P. 1987 - La Bourgogne structure positive post-hercynienne. In : Aspect et évolution géologique du bassin Parisien, Mémoire hors-série nº 6 du Bulletin d'Information des Géologues du Bassin de Paris, pp. 143-165.

ROCHE J., RIBEIRO L. et VAULTIER M. 1968 - L'industrie du gisement d'Évoramonte (Alentejo). “O Arqueólogo Português", 3osérie, 2 , pp. 7 - 13.

SMITH P. E. L. 1966 - Le Solutréen en France, Bordeaux : Imprimerie Delmas, Publication de l’Institut de Préhistoire de l'Université de Bordeaux I, mémoire nº5, 452 p.

SURMELY F., BARRIER P., BRACCO J.-P. CHARLY N. et LIABEUF R. 1998 - Caractérisation du silex par l'analyse des microfaciès et application au peuplement préhistorique de l'Auvergne (France). C.R. Acad. Sciences Paris, Sciences de la terre et des planètes, pp. 595 - 601. 
SURMELY F., LIÉGARD S., FOURVEL A., ALIX P., JAUTÉE E. et RUÉ M., 2002 - Contribution à l'étude de la circulation de longue distance des matières premières au Paléolithique. Les nucléus mis en forme découverts le long de la vallée de la Loire (Départements de l'Allier, de la Saône-et-loire et de la Loire). Paléo n 14, pp. 265 - 274.

THEVENOT J.-P. 1991 - Remarques préliminaires sur l'industrie lithique des niveaux chasséens de Chassey (La Redoute). Identité du Chasséen, Mémoire du Musée de Préhistoire de Nemours, 4, pp. $251-259$.

\section{NOTES}

1. Nous tenons à remercier Louis Bonnamour, Conservateur au Musée Denon de Chalon-surSaône, pour nous avoir facilité l'accès aux pièces et pour ses précieuses informations.

2. Le nombre exact des feuilles de laurier découvertes à Volgu reste incertain. F. Chabas mentionne 14 pièces dont 3 brisées. J.-B. Jost porte ce nombre à 17 (Jost 1927). Ce dernier auteur possédait, personnellement un fragment distal de $20 \mathrm{~cm}$, d'une d'entre elles. Mais à sa mort, la majeure partie de sa collection fut dispersée et perdue. Aujourd'hui, 15 feuilles de Volgu sont officiellement connues (16 si l'on compte le fragment ayant appartenu à J.-B. Jost, qui est indépendant des deux fragments présentés au musée Denon). Il est possible qu'il en existe d'autres entières ou fragmentées dans des collections privées, qui ne peuvent donc avoir été prises en compte dans notre étude.

\section{RÉSUMÉS}

L'étude des feuilles de laurier solutréennes de Volgu nous a permis de mettre en évidence que plusieurs microfaciès siliceux, formés dans des craies, ont servi à leur fabrication. Leur comparaison avec des échantillons géologiques collectés sur un vaste secteur géographique indique que les sources de ces matières premières se situeraient à plus de 150 kilomètres du lieu de leur découverte. Cette constatation et la comparaison avec les modes d'exploitation d'autres sources de silex du Crétacé supérieur servent de départ à une discussion sur le mode de diffusion de cette catégorie d'objets solutréens.

The study of Volgu Laurel leaf points has revealed the use of distinct sedimentary siliceous microfacies, all formed in marine chalk formations. Comparison with geological samples collected in a large geographical area permits to establish that the potential sources are distant of more than $150 \mathrm{~km}$ from Volgu. This data and the comparison with the exploitation of other upper Cretaceous sources permit to initiate a discussion about circulation modalities for this kind of Solutrean tool.

\section{INDEX}

Mots-clés : Solutréen, feuille de laurier, transport de matière première

Keywords : Solutrean, laurel leaf points, raw material transportation 


\section{AUTEURS}

\section{THIERRY AUBRY}

Ministère de la culture du Portugal, Rua da Fontinha nº 62, 3040-168 Coimbra

JEAN-BAPTISTE PEYROUSE

Lycée militaire d'Autun, 27 bis avenue Charles de Gaulle, 71400 Autun

BERTRAND WALTER

Musée de la Poterne (Preuilly-sur-Claise), Les Chirons, 37290 Preuilly-sur-Claise 